\title{
How Well Can We Predict Hypernyms from Word Embeddings? A Dataset-Centric Analysis
}

\author{
V. Ivan Sanchez Carmona and Sebastian Riedel \\ University College London \\ Department of Computer Science \\ \{i.sanchezcarmona, s.riedel\}@cs.ucl.ac.uk
}

\begin{abstract}
One key property of word embeddings currently under study is their capacity to encode hypernymy. Previous works have used supervised models to recover hypernymy structures from embeddings. However, the overall results do not clearly show how well we can recover such structures. We conduct the first dataset-centric analysis that shows how only the Baroni dataset provides consistent results. We empirically show that a possible reason for its good performance is its alignment to dimensions specific of hypernymy: generality and similarity.
\end{abstract}

\section{Introduction}

Word embeddings have been widely used as features in NLP tasks like parsing and textual entailment. One key aspect that has been investigated is their capacity to encode hypernymy; this semantic relation denotes a taxonomical order of objects in the world; for example, a dog is a canine which is $a$ vertebrate. To test the ability of embeddings to encode hypernymy, previous work has proposed supervised models to learn whether a given pair of embeddings $\left(w_{i}, w_{j}\right)$ are in the hypernymy relation (Roller et al., 2014; Necsulescu et al., 2015; Fu et al., 2014).

Results from previous work suggest that word embeddings indeed capture hypernymy information. This observation is relatively general and robust across several choices of datasets, models and embeddings. For example, Levy et al. (2015) achieve up to 0.85 F1, while Roller and Erk (2016) achieve up to $0.90 \mathrm{~F} 1$. Both of these results are achieved on the Baroni dataset (Baroni et al., 2012). For most other datasets, models achieve promising scores above $0.60 \mathrm{~F} 1$ points; e.g. Roller and Erk (2016) report $0.66 \mathrm{~F} 1$ points for a linear model on the balanced Turney dataset (Turney and Mohammad, 2015).

On closer look, however, we find that the current F1-based results may be somewhat misleading. In particular, several papers report F1 scores in the higher $60 \%$ level on balanced datasets-on such datasets a baseline that predicts each pair to be in the hypernym relation already achieves $66 \%$ F1. And when calculating accuracy instead of F1 scores we observe accuracies around 50\%-60\% for state of the art models, often barely above chance level (Table 3).

There is one striking exception when it comes to accuracy results. On the Baroni dataset, accuracy is as high as $81 \%$. These observations lead us to the following questions regarding the datasets and overall results: Are the scores on the Baroni dataset high because it is an easy dataset? Or are they high because it is easier to learn hypernymy from the Baroni training set due to its design? To what extent can the Baroni dataset help us to predict hypernyms from word embeddings?

In this work we conduct the first dataset-centric analysis across 6 datasets to empirically answer the questions above. We take inspiration from the work of (Torralba and Efros, 2011) in the computer vision domain where a set of datasets are compared and biases are exposed. In the same spirit, we compare a set of datasets by evaluating the ability of models trained on such datasets to generalize to different test distributions.

We show how the Baroni dataset outperforms the other datasets. In particular, we find that models trained on Baroni's data can outperform other models even on their home turf. For example, a model trained on Baroni's data can do better on the Kotlerman (Kotlerman et al., 2010) test set than models trained on the Kotlerman training set with the same size. 
Furthermore, we show that the Baroni dataset seems to exhibit a pronounced behaviour along two dimensions known to be relevant for hypernymy: generality and similarity. This behaviour appears to be important for the success of Baroni's dataset: if we filter and resample other training datasets with respect to this behaviour, we generally achieve better results.

\section{Background}

We first give a brief overview of hypernymy detection, important findings in this domain, and then relevant work on dataset analysis.

\subsection{Supervised Hypernym Detection}

The task is posed as a binary classification problem. An instance pair is composed of two embeddings, e.g. ( $w_{\text {cat }}, w_{\text {animal }}$, positive $)$. A vector operation such as concatenation (concat) or difference (diff) is then applied to both embeddings. Vylomova et al. (2016) learned a range of semantic relations, including hypernymy, using the diff operator and achieved positive results. Roller and Erk (2016) showed that concat with a logistic regression classifier learns to extract Hearst patterns (such as, including, etc.) from distributional vectors.

Weeds et al. (2014) and Vylomova et al. (2016) described the lexical memorization phenomenon: a classifier learns that a word $w_{i}$ is hyponym of a word $w_{j}$ based on the frequency of $w_{j}$ appearing in the hypernym slot in positive pairs. In order to avoid high scores at test time due to this effect, Weeds et al. (2014) suggest having disjoint vocabularies between training and test sets.

\subsection{Dataset Analysis}

Torralba and Efros (2011) compared a set of object recognition datasets by testing each of them across different test distributions. In order to fairly compare these datasets, Torralba and Efros (2011) first eliminated some visible biases such as sample size by normalizing the datasets. In this way, other biases in the datasets were exposed such as the photographer's shooting position, or the labellers' perception, that may not be easily observable and may harm the classifier performance. Torralba and Efros (2011) concluded that some datasets are a better representation of the problem domain.

\section{Materials}

We describe both the datasets that we compare and the word embedding model that we use as features.

\subsection{Datasets}

We pick the datasets used by Levy et al. (2015) and Weeds et al. (2014) which have disjoint training and test sets.

\begin{tabular}{||c|cc||}
\hline Dataset & Size & Ratio pos/neg \\
\hline \hline Baroni & 791 & 0.97 \\
Bless & 3225 & 0.12 \\
Kotlerman & 739 & 0.45 \\
Levy & 2932 & 0.08 \\
Turney & 539 & 1.06 \\
Weeds & 2033 & 0.98 \\
\hline
\end{tabular}

Table 1: Summary of datasets.

Baroni Baroni et al. (2012) drew instance pairs from WordNet that were manually checked to discard noisy ones.

Bless The original dataset (Baroni and Lenci, 2011) contains several semantic relations. Levy et al. (2015) used the hypernymy pairs as positive instances and the pairs in all the other semantic relations as negative instances.

Kotlerman Kotlerman et al. (2010) adapted the lexical entailment dataset of (Zhitomirsky-Geffet and Dagan, 2009).

Levy From a set of entailing propositions of the form (subject, verb, object) in (Levy et al., 2014), Levy et al. (2015) extracted entailing nouns that shared two arguments to create instance pairs.

Turney Turney and Mohammad (2015) transformed the SemEval-2012 dataset (Jurgens et al., 2012) to expand from 79 to 158 semantic relations.

Weeds Weeds et al. (2014) drew instance pairs from WordNet under the constraint that none of the words in a pair must be seen in any other pair in the same role (hyponym or hypernym).

\subsection{Word Embeddings}

We pick what we believe to be one of the most representative word embedding models.

GloVe Pennington et al. (2014) designed a vector space model using a log-bilinear regression function. They learned unsupervised word embeddings from a matrix of word co-ocurrences while maintaining linear sub-structures in such space.

We do not show results on the also widely-used model of Word2 Vec since we get similar results. 


\section{Cross-test Evaluation}

We evaluate the robustness of the six datasets for generalising to different test distributions. In order to fairly compare the datasets, we follow Torralba and Efros (2011) and remove biases such as sample size and imbalance by sub-sampling with replacement and uniformly at random the training sets. We obtain 20 subsets, i.e. samples, from each of the training sets. Each sample is normalized and balanced to 400 instances. ${ }^{1}$

We learn a model for each sample using the Scikit-learn (Pedregosa et al., 2011) package and test it on all the six test sets. We try all combinations of vector operator (diff, concat) and classifier (logistic regression, SVM). Hyperparameter tuning and model selection are performed using self-validation sets. We report AUC and accuracy scores solely for the Glove embeddings of dimensionality 50 given that the results on other embedding models are quite comparable.

\subsection{Ranking Pairs: AUC ROC}

The Area Under the ROC Curve measures the ability of a classifier to rank positive instances with respect to negative ones independently of any threshold value. Unfortunately, this metric may throw an overoptimistic value under highly imbalanced data: a disproportional number of negative instances will push the positive ones higher in the ranking, while false positives will slightly affect the overall score (Zou et al., 2016). Therefore we balance the test sets using an under-sampling scheme. $^{2}$

In Table 2 we can see that, remarkably, the Baroni dataset surpasses all datasets on their own self-test sets, except for the Bless test. Interestingly, all the training sets performed better on the Baroni test set than on their self-test set (except, for the Bless dataset). This indicates both the robust generalization and superior performance of the Baroni dataset. ${ }^{3}$

We note that no training sample has overlap with any self or cross test set, except for the Weeds dataset. On the one hand, the Weeds training sam-

\footnotetext{
${ }^{1}$ We sample 200 positive instances since that is the minimum number of positives found in any of the datasets.

${ }^{2} \mathrm{We}$ also try an oversampling scheme, but the results are comparable.

${ }^{3}$ We find that the combination of SVM classifier with RBF kernel and diff vector operator gives the best performance on validation set for all the 20 samples drawn from Baroni training set.
}

ples slightly overlap with the cross-test sets. On the other hand, the Weeds test set overlaps in at least $10 \%$ of the pairs with the cross-training samples. This may influence the cross-test scores ( $\mathrm{Vy}$ lomova et al., 2016).

\subsection{Detecting Hypernyms: Accuracy}

We optimize a threshold, on self-validation sets, for each model in Section 4.1. In Table 3 we can see again the superior performance of the Baroni dataset. While the mean of all the self-test scores (main diagonal) is 0.606 points, Baroni achieves a mean of 0.655 points.

Interestingly, in average all the datasets perform close to a random behavior, with the exception of the Baroni and Weeds datasets. ${ }^{4}$ Furthemore, this poor behavior is observed on self-test sets for 3 datasets (Kotlerman, Levy, and Turney). This contrasts to the AUC scores obtained before. One possible cause may be a sensitivity problem in the threshold optimization.

\section{Dataset Analysis}

We provide an empirical rationale behind the good performance of the Baroni dataset: we believe it aligns to two dimensions specific of hypernymy generality and similarity-i.e. the instances in the dataset form what we believe to be patterns denoting hypernymy. We explain below these patterns.

We use WordNet (Fellbaum, 1998) to compute both generality and similarity levels. We define generality levels as the absolute difference, in number of edges, of two words to the root of the taxonomy: $g=\mid$ distance $\left(\right.$ wor $_{1}$, root $)-$ distance $\left(\right.$ wor $_{2}$, root $) \mid$. We define similarity levels as the similarity score between two words; we use the Wu-Palmer function. ${ }^{5}$

We explain now the patterns mentioned above. In the generality level $g=0$, where co-hyponyms exist, we expect only negative pairs to populate the dataset. In the rest of the levels, we would expect a distribution where the number of instance pairs is inversely proportional to the generality level because the branching factor at the bottom levels is greater by a factor $\alpha$ in comparison to the top levels; this means that we are more likely to sample pairs of words connected by fewer number of

\footnotetext{
${ }^{4}$ However, recall that as noted in Sec. 4.1, Weeds scores on cross-test results may be influenced by lexical memorization issues.

${ }^{5}$ We re-scale from $[0.0,1.0]$ to $[-1.0,1.0]$ for visualization purposes.
} 


\begin{tabular}{|c|c|c|c|c|c|c|c|}
\hline Train Test & Baroni & Bless & Kotlerman & Levy & Turney & Weeds & Mean \\
\hline$\overline{\text { Baroni }}$ & 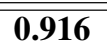 & 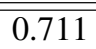 & 0.616 & 0.702 & 0.654 & 0.686 & 0.714 \\
\hline Bless & 0.762 & 0.850 & 0.555 & 0.632 & 0.600 & 0.615 & 0.669 \\
\hline Kotlerman & 0.653 & 0.612 & 0.543 & 0.566 & 0.581 & 0.544 & 0.583 \\
\hline Levy & 0.716 & 0.611 & 0.592 & 0.698 & 0.569 & 0.533 & 0.619 \\
\hline Turney & 0.686 & 0.646 & 0.547 & 0.595 & 0.646 & 0.520 & 0.606 \\
\hline Weeds & 0.817 & 0.645 & 0.574 & 0.687 & 0.637 & 0.675 & 0.672 \\
\hline
\end{tabular}

Table 2: Cross-test performance: Mean AUC scores over 20 samples. Self-test score in bold.

\begin{tabular}{|c|c|c|c|c|c|c|c|}
\hline Train Test & Baroni & Bless & Kotlerman & Levy & Turney & Weeds & Mean \\
\hline Baroni & 0.812 & 0.638 & 0.587 & 0.653 & 0.60 & 0.636 & 0.655 \\
\hline Bless & 0.578 & 0.642 & 0.505 & 0.526 & 0.524 & 0.508 & 0.547 \\
\hline Kotlerman & 0.563 & 0.546 & 0.520 & 0.524 & 0.528 & 0.528 & 0.534 \\
\hline Levy & 0.521 & 0.510 & 0.507 & 0.522 & 0.509 & 0.496 & 0.510 \\
\hline Turney & 0.546 & 0.534 & 0.518 & 0.540 & 0.540 & 0.479 & 0.526 \\
\hline Weeds & 0.736 & 0.579 & 0.553 & 0.626 & 0.599 & 0.600 & 0.615 \\
\hline
\end{tabular}

Table 3: Cross-test performance: Mean accuracy scores over 20 samples. Self-test score in bold.

edges than by higher number of edges.

On the other hand, for the similarity distribution, as a function of the number of edges, at large values we expect a dominance of positive instances because the number of edges between the words in a true hypernym pair is generally fewer than between a non-hypernym pair. In addition, as we argued for the generality distribution, we are more likely to sample shorter hypernym pairs than longer pairs.

\subsection{Exploring the Baroni dataset}

In Fig. 1 we see that at level $g=0$ only negative pairs are found in the Baroni dataset. We also observe that the distribution matches the expected distribution along generality levels. In Fig. 2 we see that from the level $s=0.2$, towards the highest levels, there is a clear dominance of positive pairs; though we also find negative pairs in these levels. These negative pairs may be positive pairs reversed, e.g. ( $w_{\text {animal }}, w_{\text {cat }}$, negative), or pairs with related words, e.g. ( $w_{\text {cat }}, w_{\text {invertebrate }}$, negative). We also see that from the level $s=0.1$ towards the lowest levels, the negative pairs dominate.

We compare the Baroni distribution with the Turney distribution. In Fig. 3 we observe that the shape of the generality distribution roughly fits our expected distribution; however, we see that positive pairs populate level $g=0$. This seems to show that around $10 \%$ of the positive pairs in the
Turney dataset are spurious pairs.

In Fig. 4 we observe that the similarity distribution from the Turney dataset does not fit the expected distribution. Even though at high levels the dominance is mainly of positive pairs, at low levels we also see a strong presence of positive pairs along with negative pairs. This may imply that a high number of positive pairs are noisy or inconsistent, which may explain the low performance of the Turney dataset.

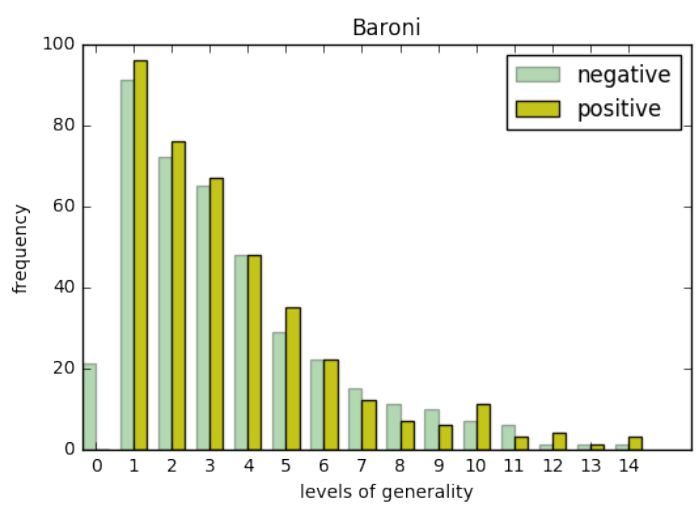

Figure 1: Distribution of instance pairs on the Baroni dataset along generality levels.

\subsection{Mimicking the Baroni Distribution}

We believe that the patterns found in the Baroni training set may be part of the cause of its good performance. To corroborate our hypothesis, we draw a new training set from the union of all the 


\begin{tabular}{|c|c|c|c|c|c|c|c|}
\hline Train $\quad$ Test & Baroni & Bless & Kotlerman & Levy & Turney & Weeds & Mean \\
\hline New train set & $0.794(0.05)$ & $0.664(0.02)$ & $0.580(0.03)$ & $0.644(0.02)$ & $0.596(0.02)$ & $0.629(0.03)$ & 0.651 \\
\hline Baseline & $0.775(0.06)$ & $0.655(0.02)$ & $0.566(0.03)$ & $0.641(0.02)$ & $0.596(0.02)$ & $0.598(0.03)$ & 0.638 \\
\hline
\end{tabular}

Table 4: New dataset vs. Baseline: Mean accuracy scores and standard deviation over 20 samples.

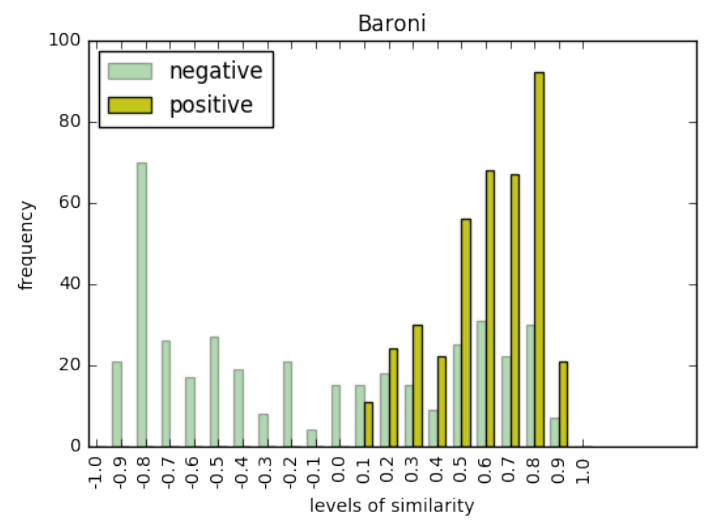

Figure 2: Distribution of instance pairs on the Baroni dataset along similarity levels.

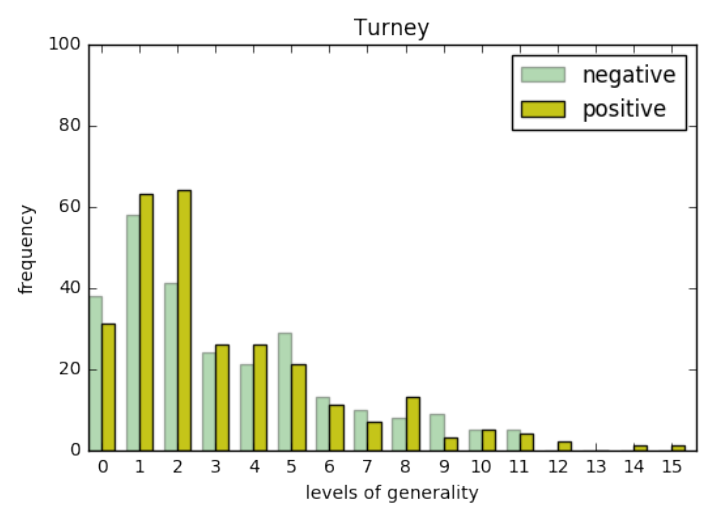

Figure 3: Distribution of instance pairs on the Turney dataset along generality levels.

training sets such that we mimic the Baroni distributions in Fig. 1 and Fig. 2. More specifically, we allow a pair to populate our new training set if it fulfils constraints regarding the number of instances along generality and similarity levels.

One example constraint that needs to be fulfilled for positive pairs is: IF generality level $g>0$ AND positive vs. negative pairs ratio is fulfilled according to ratio $r_{g}$ AND similarity level $s>=$ 0.1 AND positive vs. negative pairs ratio is fulfilled according to ratio $r_{s}$ THEN accept pair.

We obtain 20 balanced and normalized samples populated with 400 instances in each of them. We compare against a dataset baseline where we allow any pair, chosen uniformly at random, to populate

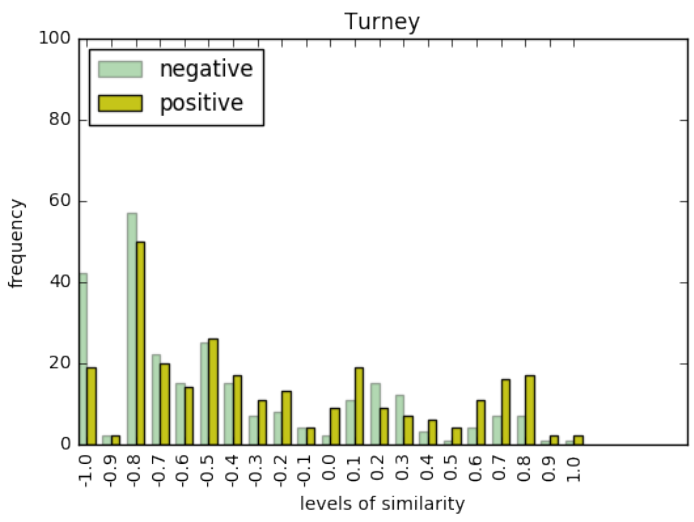

Figure 4: Distribution of instance pairs on the Turney dataset along similarity levels.

the baseline. For building the dataset baseline, we use the same random seeds as those used for building the samples that mimic the Baroni distribution. In Table 4 we see how the new training set robustly outperforms the baseline. These results support our hypothesis for why the Baroni dataset is able to outperform all the datasets.

\section{Conclusions}

We performed the first dataset-centric analysis for investigating how well we can predict hypernym pairs from word embeddings. We showed in crosstest evaluations how -in contrast to what results from previous work suggest- the Baroni dataset is the only one that consistently enables us to predict hypernym pairs. We empirically showed that the superior performance of the Baroni dataset may be in part due to its alignment to two dimensions relevant to of hypernymy: generality and similarity. We empirically corroborated this hypothesis by building a new training set that mimics the Baroni distribution and outperforms on average a dataset baseline.

\section{Acknowledgments}

The first author was sponsored by CONACYT. The second author was supported by an Allen Distinguished Investigator Award and a Marie Curie Career Integration Award. 


\section{References}

Marco Baroni and Alessandro Lenci. 2011. How we blessed distributional semantic evaluation. In Proceedings of the GEMS 2011 Workshop on GEometrical Models of Natural Language Semantics, pages 1-10. Association for Computational Linguistics.

Marco Baroni, Raffaella Bernardi, Ngoc-Quynh Do, and Chung-chieh Shan. 2012. Entailment above the word level in distributional semantics. In Proceedings of the 13th Conference of the European Chapter of the Association for Computational Linguistics, pages 23-32, Avignon, France, April. Association for Computational Linguistics.

Christiane Fellbaum. 1998. WordNet: An Electronic Lexical Database. MIT Press., Cambridge, MA.

Ruiji Fu, Jiang Guo, Bing Qin, Wanxiang Che, Haifeng Wang, and Ting Liu. 2014. Learning semantic hierarchies via word embeddings. In Proceedings of the 52nd Annual Meeting of the Association for Computational Linguistics (Volume 1: Long Papers), pages 1199-1209, Baltimore, Maryland, June. Association for Computational Linguistics.

David Jurgens, Saif Mohammad, Peter Turney, and Keith Holyoak. 2012. Semeval-2012 task 2: Measuring degrees of relational similarity. In *SEM 2012: The First Joint Conference on Lexical and Computational Semantics - Volume 1: Proceedings of the main conference and the shared task, and Volume 2: Proceedings of the Sixth International Workshop on Semantic Evaluation (SemEval 2012), pages 356-364, Montréal, Canada, 7-8 June. Association for Computational Linguistics.

Lili Kotlerman, Ido Dagan, Idan Szpektor, and Maayan Zhitomirsky-Geffet. 2010. Directional distributional similarity for lexical inference. Natural Language Engineering, 16(04):359-389.

Omer Levy, Ido Dagan, and Jacob Goldberger. 2014. Focused entailment graphs for open ie propositions. In Proceedings of the Eighteenth Conference on Computational Natural Language Learning, pages 87-97, Ann Arbor, Michigan, June. Association for Computational Linguistics.

Omer Levy, Steffen Remus, Chris Biemann, and Ido Dagan. 2015. Do supervised distributional methods really learn lexical inference relations? In Proceedings of the 2015 Conference of the North American Chapter of the Association for Computational Linguistics: Human Language Technologies, pages 970-976, Denver, Colorado, May-June. Association for Computational Linguistics.

Silvia Necsulescu, Sara Mendes, David Jurgens, Núria Bel, and Roberto Navigli. 2015. Reading between the lines: Overcoming data sparsity for accurate classification of lexical relationships. In Proceedings of the Fourth Joint Conference on Lexical and Computational Semantics, pages 182-192, Denver,
Colorado, June. Association for Computational Linguistics.

F. Pedregosa, G. Varoquaux, A. Gramfort, V. Michel, B. Thirion, O. Grisel, M. Blondel, P. Prettenhofer, R. Weiss, V. Dubourg, J. Vanderplas, A. Passos, D. Cournapeau, M. Brucher, M. Perrot, and E. Duchesnay. 2011. Scikit-learn: Machine learning in Python. Journal of Machine Learning Research, 12:2825-2830.

Jeffrey Pennington, Richard Socher, and Christopher Manning. 2014. Glove: Global vectors for word representation. In Proceedings of the 2014 Conference on Empirical Methods in Natural Language Processing (EMNLP), pages 1532-1543, Doha, Qatar, October. Association for Computational Linguistics.

Stephen Roller and Katrin Erk. 2016. Relations such as hypernymy: Identifying and exploiting hearst patterns in distributional vectors for lexical entailment. In Proceedings of the 2016 Conference on Empirical Methods in Natural Language Processing, pages 2163-2172, Austin, Texas, November. Association for Computational Linguistics.

Stephen Roller, Katrin Erk, and Gemma Boleda. 2014. Inclusive yet selective: Supervised distributional hypernymy detection. In Proceedings of COLING 2014, the 25th International Conference on Computational Linguistics: Technical Papers, pages 10251036, Dublin, Ireland, August. Dublin City University and Association for Computational Linguistics.

Antonio Torralba and Alexei A. Efros. 2011. Unbiased look at dataset bias. In Computer Vision and Pattern Recognition (CVPR), 2011 IEEE Conference on, pages 1521-1528. IEEE.

Peter D. Turney and Saif M. Mohammad. 2015. Experiments with three approaches to recognizing lexical entailment. Natural Language Engineering, 21(03):437-476.

Ekaterina Vylomova, Laura Rimell, Trevor Cohn, and Timothy Baldwin. 2016. Take and took, gaggle and goose, book and read: Evaluating the utility of vector differences for lexical relation learning. In Proceedings of the 54th Annual Meeting of the Association for Computational Linguistics (Volume 1: Long Papers), pages 1671-1682, Berlin, Germany, August. Association for Computational Linguistics.

Julie Weeds, Daoud Clarke, Jeremy Reffin, David Weir, and Bill Keller. 2014. Learning to distinguish hypernyms and co-hyponyms. In Proceedings of COLING 2014, the 25th International Conference on Computational Linguistics: Technical Papers, pages 2249-2259, Dublin, Ireland, August. Dublin City University and Association for Computational Linguistics.

Maayan Zhitomirsky-Geffet and Ido Dagan. 2009. Bootstrapping distributional feature vector quality. Computational linguistics, 35(3):435-461. 
Quan Zou, Sifa Xie, Ziyu Lin, Meihong Wu, and Ying Ju. 2016. Finding the best classification threshold in imbalanced classification. Big Data Research. 